\title{
Carnets
}

Revue électronique d'études françaises de l'APEF

Deuxième série - 5 | 2015

Imaginaires de guerre et autres conflits

\section{Guerre totale et guerre sacrée}

Les métamorphoses de la Grande Guerre dans La Chanson de Vaux-

Douaumont

\section{Olivier Wicky}

\section{OpenEdition}

9 Journals

Édition électronique

URL : http://journals.openedition.org/carnets/343

DOI : 10.4000/carnets.343

ISSN : 1646-7698

Éditeur

APEF

Référence électronique

Olivier Wicky, "Guerre totale et guerre sacrée », Carnets [En ligne], Deuxième série - 5 | 2015, mis en ligne le 30 novembre 2015, consulté le 19 avril 2019. URL : http://journals.openedition.org/ carnets/343; DOI : 10.4000/carnets.343

Ce document a été généré automatiquement le 19 avril 2019

\section{(c) (i) (8)}

Carnets est mis à disposition selon les termes de la licence Creative Commons - Atribution - Pas d'utilisation commerciale 4.0 International. 


\title{
Guerre totale et guerre sacrée
}

\author{
Les métamorphoses de la Grande Guerre dans La Chanson de Vaux- \\ Douaumont
}

Olivier Wicky

1 Écrivain catholique parfois mal considéré par la censure ecclésiastique, chantre de la terre savoyarde mais grand voyageur, académicien nourrissant une vaste correspondance avec les figures majeures de son temps mais presque oublié de nos jours : le profil d'Henry Bordeaux a de quoi surprendre par sa richesse et sa complexité. Ce polygraphe prolifique - dont les convictions sont souvent rapprochées de celles des fameux auteurs « en B » de la littérature conservatrice (Barrès, Bourget, Bazin...) - a laissé derrière lui une œuvre immense, riche de plus de 250 titres, dont d'innombrables romans, plusieurs études critiques et historiques ainsi que 13 volumes de mémoires, consacrant le romancier et essayiste savoyard comme un des acteurs majeurs du champ littéraire de la première moitié du xx siècle.

2 Né en 1870, mort en 1963, Bordeaux connut également de près bien des vicissitudes historiques, dont trois guerres qui marquèrent profondément son existence :

(...) il avait quelques mois lorsque son père partit en octobre 1870 ; la dernière de ses filles avait deux ans quand il fut mobilisé ; enfin, début septembre 1939, il tenait sur ses genoux un petit-fils de neuf mois dont le père venait de partir, comme lieutenant d'artillerie. (Buttin, $1990: 40$ )

C'est donc durant la Première Guerre mondiale que Bordeaux mit parallèlement au service de la France ses compétences militaires et ses talents littéraires: officier dans l'armée territoriale en raison d'une blessure, il va en effet demander à être versé dans l'armée active et sera nommé "officier de liaison à Verdun, chargé de rédiger un historique destiné aux ambassades, missions à l'étranger et à l'état-major. » (Buttin, 1990 : 41). De cette expérience, Bordeaux tirera de nombreux textes, dont La Chanson de VauxDouaumont, récit en deux volumes consacré aux combats qui se déroulèrent dans la Meuse pour le contrôle de différentes fortifications. Le premier tome, intitulé Les derniers jours $d u$ fort de Vaux, dédicacé "Aux soldats de Verdun", relate la perte des forts de Vaux et Douaumont, pris respectivement par les Allemands le 25 février et le 7 juin 1916; le 
second volet, Les Captifs délivrés - cette fois dédicacé au Général Pétain ${ }^{1}$ - raconte quant à lui la reprise de ces édifices, sur une période allant du 21 octobre au 3 novembre 1916.

Enrichis de cartes, de lettres et de rapports divers, fournissant une description minutieuse des événements, ces deux documents représentent un témoignage d'exception aussi bien d'un point de vue factuel qu'idéologique, de nombreuses considérations historiques, politiques et spirituelles se retrouvant au fil du récit. Bordeaux déploie en effet toute une stratégie argumentative pour donner une coloration héroïque aux combats et une dimension métaphysique à l'ensemble du conflit, faisant de la Grande Guerre une croisade destinée à briser une fois pour toutes les ambitions allemandes. Profondément blessé par la défaite de 1870, l'auteur appartient à cette génération qui, bercée par la complainte des provinces perdues et désireuse de venger Sedan, attend l'heure où la France pourra enfin laver l'affront de sa capitulation passée.

Même si les partisans de la Revanche n'ont été de 1871 à 1914 qu'une minorité selon l'historien Bertrand Joly, il ne faut pas oublier leur grande influence dans la société ni l'important soutien dont ils bénéficièrent; "le pays, si pacifique qu'il fût, leur reconnaissait malgré tout une certaine représentativité et leur déléguait tacitement une fonction protestataire et commémorative jugée indispensable » (Joly, 2012 : 150). De fait, on peut, sans trop extrapoler, considérer que les opinions développées par Bordeaux reflètent celles de toute une frange de l'armée ${ }^{2}$, de la classe politique et de la population. "Commandant, vous nous avez réconfortées dans la guerre ", diront d'ailleurs bien des années plus tard de jeunes lectrices belges à Bordeaux, expliquant que la Chanson fut saisie et brûlée par les Allemands avant de reparaître, vraisemblablement dans la clandestinité (Bordeaux, 1960 : 82). Mais alors que beaucoup de récits de guerre rejettent avec une même vigueur héroïsme et patriotisme - songeons au cri de Bardamu: « Moi, quand on me parlait de la France, je pensais irrésistiblement à mes tripes » (Céline, 2006 : 59) - La Chanson de Vaux-Douaumont s'inscrit dans une logique inverse; refusant l'absurdité de la guerre, elle cherche à transcender les souffrances en leur donnant un sens et un but de nature quasiment religieuse.

6 Mais comment se traduit cette volonté de changer cette guerre en un véritable « choc des civilisations » pour reprendre le titre du célèbre essai de Samuel Huntington? Par quels processus est-on parvenu à sacraliser le combat contre l'Allemagne, elle-même dépeinte comme une entité infernale ? Sur quels arguments s'est construite la dialectique belliciste d'une Revanche attendue pendant plus de 40 ans et enfin concrétisée ? Pour répondre à ces questions, je commencerai par analyser brièvement les liens établis par Bordeaux entre Verdun et le Moyen Âge - en particulier la Chanson de Roland - avant de m'intéresser à la façon dont il aborde le thème de la mort au combat. Enfin, je reviendrai sur l'usage de la parole de l'ennemi et les différentes manières de l'exploiter pour servir l'argumentation de l'auteur.

\section{De Roncevaux à Verdun}

7 L'idée d'associer les terribles vicissitudes de la bataille de Verdun avec le Moyen Âge semble s'être imposée de manière naturelle à plusieurs écrivains, mettant ainsi en place un véritable topos au sein de la littérature de guerre; relevons toutefois que ce lien n'implique pas forcément de connotation idéologique bien définie et que cette comparaison polysémique sera utilisée de manière aussi bien dépréciative qu'élogieuse. Par conséquent, la symbolique médiévale est tantôt exploitée pour souligner l'horreur de 
la guerre ou au contraire pour magnifier sa dimension épique. Mais sur quels éléments s'appuie ce rapprochement, alors que l'on prête volontiers un caractère éminemment moderne à la Première Guerre mondiale ? Que ce soit par la nature des armes utilisées en particulier l'artillerie ou les chars d'assaut - ou les stratégies mises en place, tout porte à penser qu'il s'agit d'un événement unique, rendu possible par les avancées technologiques de l'époque moderne et sans nul autre précédent historique. Si, comme l'affirme l'historien Jean-Yves Le Naour, «le $\mathrm{xx}^{\mathrm{e}}$ siècle était décidément un enfant de la Grande Guerre » (Le Naour, 2012: 7), l'inverse est tout aussi vrai et cette sinistre filiation peut difficilement être remise en question.

Chez Bordeaux, le rapprochement des deux périodes renvoie avant tout à une idée chevaleresque de la guerre, où le courage est tenu en haute estime et où l'on affronte un ennemi souvent supérieur par la force et le nombre mais toujours inférieur sur le plan moral.

Le titre même de ce diptyque littéraire ne plaide-t-il d'ailleurs pas en faveur d'une telle conception? En reprenant cette dénomination, Bordeaux place ces deux récits dans le vaste corpus des chansons de geste qui, de la chanson d'Antioche à celle de la croisade albigeoise, raconte les exploits des héros d'antan. Dès les premières lignes de son cycle, l'auteur précise en outre que " [1]a défense [du fort de Vaux] évoque, par delà nos siècles tout dorés de splendeur militaire, nos vieilles chansons de geste, une Chanson de Roland dont le premier personnage, invisible et toujours présent, est l'honneur français.» (Bordeaux, 1916: 3). Roland est en effet un personnage clé dans l'imaginaire collectif de la $3^{e}$ République, qui voit en lui «un mélange du Christ et de Leonidas " (Schivelbusch, 2006 : 134) : vaincu sans avoir perdu son honneur, résistant sans fléchir aux assauts, et sacrifiant enfin sa vie pour une noble cause, il incarne pleinement la France meurtrie de 1870 et ses espoirs de revanche ${ }^{3}$.

Or, si Bordeaux compare volontiers le défunt guerrier aux forts captifs, il va plutôt se concentrer sur la figure de l'empereur et c'est d'ailleurs dans le chapitre intitulé Les trompettes de Charlemagne qu'il décrira de façon détaillée le lien unissant Roncevaux à Verdun. Il ne s'agit pas ici de simples allusions : à plusieurs reprises, il cite le texte original de la Chanson de Roland - dont l'auteur est appelé affectueusement «le vieux poète » - et mêle étroitement les deux récits dans un même souffle épique. Néanmoins, ce n'est pas l'agonie superbe de Roland qui va retenir son attention, mais bien plutôt la « sainte tâche des représailles » (Bordeaux : 1917, 40), puisque selon lui, la délivrance des deux forts « évoque le retour de Charlemagne sur le champ de bataille de Roncevaux et la vengeance de l'empereur » (39). Et pour l'accomplir, les Français pourront compter sur "plus de 600 bouches à feu » (41) qui, à l'image des trompettes de Charlemagne, vont annoncer aux Allemands l'imminence de leur défaite: nous sommes ici bien loin des réceptions romantiques $\mathrm{du}$ xix ${ }^{\mathrm{e}}$ siècle, comme le fameux poème du Cor de Vigny qui ne s'attachait qu'à la mort tragique du héros sans guère se soucier du châtiment des Maures ! Mais ce parallélisme implique aussi une similitude dans les rangs adverses : les Allemands sont par conséquent confondus avec de nouveaux Sarrasins, antagonistes héréditaires de la chrétienté et de l'Occident. Avec ces références à l'épopée de Roland, un premier pas est donc franchi vers la diabolisation de l'ennemi. 


\section{La mort transfigurée}

12 On remarque cependant que l'association entre Grande Guerre et période médiévale est aussi souvent liée aux aspects les plus tragiques du conflit. Devant l'ampleur des carnages, les témoins sont ainsi tentés d'évoquer le Moyen Âge, qui reste en ce début de siècle perçu comme une période sombre et sanglante ${ }^{4}$. Certes, ce genre de réflexion repose bien souvent sur une vision fantasmée de l'époque, presque entièrement réduite à sa part de noirceur, mais ne manque néanmoins pas de pertinence, ne serait-ce qu'en ce qui concerne le rapport du soldat à la mort, devenue compagne familière en raison des nouvelles règles adoptées par les belligérants :

Il est par ailleurs impossible aux combattants en ligne d'échapper aux visions macabres, celles des corps gisant dans le no man's land ou sur les "séchoirs " [barbelés], ou encore celles des dépouilles qui ont été sommairement enterrées et exhumées par des obus. (...)

Si de nombreux tués gisent ainsi entre les lignes pendant des semaines ou des mois, c'est que, contrairement à la plupart des guerres de siège du passé, les trêves pour le ramassage et l'ensevelissement des morts - qu'elles soient improvisées ou organisées - ne sont plus que très rarement observées. (Hardier, 2012 : 352-353)

Dans de telles conditions, il n'est guère étonnant que les vivants soient durement confrontés à leur statut de mortels et comme le confirme l'historien Thierry Hardier : «(...) ces spectacles macabres marquent les esprits; ces cadavres, de façon spéculaire, renvoient ceux qui les regardent à leur propre fin. » (Hardier, 2012: 352). Par le biais de ce memento mori imposé, le poilu de 14-18 renoue ainsi avec une pensée paradigmatique courante au Moyen Âge, où la représentation - souvent repoussante - de la Faucheuse ou du cadavre pourrissant sert de prétexte à une introspection et à une confrontation avec l'imminence de son propre trépas. La mort, rendue lointaine par les progrès de la médecine et le recul des croyances religieuses apparait soudain comme une réalité glaçante qui ne peut que renvoyer au Moyen Âge dont les artistes et poètes n'hésitaient pas à représenter la décomposition des chairs avec une dérangeante précision. Avec la Grande Guerre, c'est en quelque sorte le retour de ces danses macabres, courantes entre le XIV et le $\mathrm{XV}^{\mathrm{e}}$ siècle, où des corps putréfiés emmènent à leur suite toutes les classes de la population pour mieux symboliser l'empire absolu de la mort :

Quoique les circonstances permettent parfois d'éviter ces affreuses exhibitions, les procédures d'ensevelissement n'en restent pas moins inhabituelles et effrayantes: en effet, "l'emploi des fosses communes pouvant contenir jusqu'à 100 corps, rappelant les mesures prises lors des grandes épidémies du Moyen Âge et de l'époque moderne, est prescrit par le GQG français dès le début de la guerre. » (Hardier, 2012 : 356-357)

14 Non seulement la guerre rend la mort habituelle, mais elle bouleverse profondément les rapports que l'homme entretient avec elle. Le respect qui l'entoure cède le pas à une familiarité grossière tandis que la peur inspirée se combat tant bien que mal par un cynisme morbide. Ainsi, dans Prélude à Verdun, Jules Romains prête à un de ses personnages, Jerphanion, le discours suivant, où les ébranlements de la morale se confondent avec une série de lieux communs sur la période médiévale :

Moi je vois plutôt ça dans le style Moyen Âge. Nous sommes en train de retrouver des états d'esprit du Moyen Âge : l'irrévérence pour la carcasse humaine et pour la chair vivante ou morte; l'énorme place qu'elles tiennent pourtant dans les préoccupations; la complaisance ricanante pour les aspects où le corps se dégrade, 
comme l'excrément, la charogne, le squelette ; le côté danse macabre, ossuaire, gargouilles, bas-reliefs obscènes, latrines qui fermentent à l'ombre de la cathédrale, des grands siècles chrétiens. (Romains, 1958 : 106) guerre de Blaise Cendrars, une évocation quelque peu similaire, où l'idée de mort réactive aussi le topos médiéval; mais au lieu d'un voisinage direct, c'est plutôt d'une mort intériorisée qu'il s'agit, inscrite dans les souvenirs de l'auteur et où les disparus de la Première Guerre mondiale viennent se confondre avec ceux de la Seconde :

La mémoire, quel cimetière ! Proches ou lointaines, les tombes se multiplient et dans une époque comme la nôtre, les morts jouent à saute-mouton et reviennent, fonçant des cieux ! Le Pilote de la Mort. Ce n'est pas un film, mais le prototype d'un nouveau Moyen Âge. Quelle fresque !... (Cendrars, $1946: 68$ )

De son côté, Henry Bordeaux n'échappe évidemment pas à cette omniprésence de la mort, pas plus qu'au memento mori suscité par son spectacle, mais le récit qu'il en tire reste néanmoins assez éloigné des habituels saisissements d'effroi ou de l'indifférence qui marque parfois ces rencontres d'outre-tombe. Si elles sont pour lui une parenthèse dans la description des combats et l'occasion d'un discours moral, ce n'est pas pour dénoncer la guerre - comme on peut le voir chez Barbusse, Cendrars ou encore Céline -, mais plutôt pour la transcender et lui donner un sens vertueux. D'ailleurs, la haute estime dans laquelle il tient le Moyen Âge ${ }^{5}$ l'empêche d'avoir recours à cette période pour caractériser l'hécatombe, même, si, comme nous allons le voir, ses commentaires sont profondément liés à une conception médiévale de la mort. Un épisode en particulier, explicitement intitulé Méditation sur la mort, occupe un chapitre entier des Derniers jours du Fort de Vaux, et prouve bien l'importance de cette réflexion dans sa démarche narrative.

Le passage en question, daté du 18 mars 1916, s'ouvre par un moment de repos, pris sur une "colline qui domine Verdun", par un «triomphal soir de printemps » (Bordeaux, 1916 : 113). L'auteur, étonné par le contraste entre le paysage paisible et la guerre qui y fait pourtant rage, décide de s'allonger un moment dans l'herbe avant de voir qu'un autre soldat se trouve près de lui « couché de tout son long » et ne " prêtant pas attention à [s]a venue ». Mais Bordeaux ne tarde pas à faire une tragique constatation, qui ne va pas sans rappeler la fin du Dormeur du Val de Rimbaud: "Je le regarde mieux: sa figure n'est qu'une plaie. Je m'approche : c'est un mort. On ne vient pas ici pour s'isoler et rêver. Rien ne s'accomplit ici que sous le manteau de la mort. »(Bordeaux, 1916 : 114).

La différence avec d'autres rencontres du même type dans la littérature de guerre est on ne peut plus flagrante: le cadavre est appréhendé de manière si détachée et lointaine qu'on aurait pu le prendre pour un homme endormi mais bel et bien vivant. Il est vrai que la blessure est évoquée - l'auteur suggère même qu'elle est effroyable - mais au lieu de s'attarder à sa description, il choisit d'évacuer tout le pathos de la scène pour se concentrer sur sa signification et amorcer la méditation annoncée par le titre. Là encore, s'il commence par reprendre le topos de l'incessante proximité du trépas - « Mais la mort 
avec la guerre a perdu de son importance. Elle est devenue familière. » (Bordeaux, 1916 : 114), ou encore " Oui, chacun s'est fait à l'idée de la mort (115) »-, il opère tout de même une distinction entre ce qu'il qualifie de mort «non glorieuse », c'est-à-dire celle qui est causée par les obus, celle qui est la conséquence d'une action hasardeuse ou anonyme de l'ennemi et n'implique pas de contact direct avec celui-ci, et la mort courageuse, illustrée quelques lignes plus loin avec l'exemple du capitaine D.... Cette antinomie entre deux types de décès était elle aussi fortement enracinée dans la pensée médiévale, où bonne mort et male mort symbolisaient les deux pôles d'une issue fatale, susceptibles de conditionner toute vie dans l'au-delà.

Si Bordeaux admet que la mort «sournoise et effroyable sous la forme d'un bloc de fer lancé à des kilomètres de distance (...) inspire le plus profond dégoût » (Bordeaux, 1916 : 114-115), il estime aussi qu'une certaine abnégation permet d'en atténuer l'horreur, voir même de la transcender totalement :

Si l'on ne se révolte pas contre elle, si on consent à l'accepter, voici qu'elle se transforme à la façon des sorcières de jadis dans les contes de fées. Le hideux squelette se recouvre de jeunes chairs qui sentent les fleurs. Le visage qu'elle approche est d'une beauté lumineuse. Dans le baiser qu'elle donne, passe la tendresse de la Patrie pour ses enfants. (Bordeaux, 1916 : 114-115)

21 Cet adoucissement de la mort, cette quasi érotisation reprend elle aussi un concept médiéval récurrent, que l'on retrouve par exemple dans les Lais de Marie de France et que Laurent Guyénot, dans son ouvrage sur la mort féerique, qualifie de "noces funèbres " (Guyénot, 2011 : 147) : aux derniers instants, une figure féminine, le plus souvent une fée, permet au mourant de rejoindre un autre horizon, séjour enchanteur assimilé aux Îles Fortunées ou à Avalon. Ces «figures psychopompes (...) incarnent la belle mort, vue comme une union amoureuse selon un symbolisme que connaissent bien toutes les traditions mystiques.» (Guyénot, 2011: 147). Et dans la mystique patriotique de Bordeaux, l'acquiescement serein à l'arrêt du destin permet également un passage vers un autre monde - certes peu détaillé mais sans doute paradisiaque au vu des rares détails de cette métamorphose - tandis que le couple Mort-Patrie se substitue à l'image de la fée et devient l'amante du soldat ayant consenti au sacrifice suprême.

Avec cette première description d'une mort brutale et vaine, Bordeaux développe un point de vue radicalement opposé à celui d'autres écrivains confrontés aux cadavres de leurs frères d'armes. Prenons par exemple le cas d'Henri Barbusse : dans Le Feu, il raconte la pénible découverte d'un corps avec de puissants accents naturalistes propres à susciter un profond sentiment de dégoût chez le lecteur; la scène s'ouvre d'ailleurs par un échange qui illustre l'incapacité de distinguer quoi que ce soit d'humain dans le trépassé :

- Je ne vois rien. La vue est bouchée. Qu'est-ce que c'est que ce paquet d'étoffes?

- C'est lui, dit Paradis.

Ah! c'était un cadavre, un cadavre assis dans un trou, épouvantablement proche.

(...)

Reconnaissable, malgré ses yeux exorbités et opaques qui louchaient, le bloc de sa barbe vaseuse et sa bouche tordue qui montrait les dents. Il avait l'air, à la fois, de sourire et de grimacer à son fusil, embourbé, debout, devant lui. Ses mains tendues en avant étaient toutes bleues dessus et écarlates en dessous, empourprées par un humide reflet d'enfer. C'était lui, lavé de pluie, pétri de boue et d'une espèce d'écume, souillé et horriblement pâle, mort depuis quatre jours tout contre notre talus, que le trou d'obus où il était terré avait entamé. (Barbusse, 1917 : 235)

23 Altération des formes et des couleurs, déformations grotesques, abandon du corps aux éléments et stupeur de la macabre découverte: tels sont les arguments utilisés par 
Barbusse pour souligner la vanité de la mort au combat et faire avorter toute tentative de glorification patriotique. À l'inverse de Bordeaux qui pense d'abord que le soldat est endormi, le narrateur de Barbusse ne distingue même pas de forme humaine et là où le premier croit discerner la vie, l'autre n'est même plus capable de voir la mort, mais estime se trouver devant un simple paquet d'étoffes. Relevons aussi que le texte de Bordeaux, de par son caractère officiel et sa vocation patriotique, ne doit pas démoraliser ses lecteurs et souscrit donc à une forme de discours contraint, au sens où l'entend Jean Kaempfer dans sa Poétique du récit de guerre :

Autre reniement, dicté, celui-là, par la charité : aux familles qui écrivent "pour avoir des détails sur la mort d'un soldat", on offre systématiquement la représentation standard d'une fin digne et consolante - la mort instantanée, une balle en plein cœur ou au milieu du front... (Kaempfer, 1998 : 242)

Qu'en est-il alors de la mort glorieuse, celle que l'on reçoit au combat? Bordeaux donne cette fois la parole au capitaine D..., blessé deux fois à Verdun, qui narre son ressenti à l'instant crucial où il gît à terre, touché à la poitrine :

Je pensais que j'allais mourir et j'étais dans un état de joie infinie. Mon amour pour ma femme et mon fils que je devais quitter n'en était nullement altéré. Je ne sais comment vous expliquer : rien ne me pesait plus et de mes plus chers sentiments je me sentais délivré. Comment rencontrerais-je jamais pareille occasion de mourir? Tout, en moi, autour de moi, était léger, facile comme un vol d'oiseau. Je ne souffrais plus. Dans ma difficulté à respirer même, je trouvais une sorte de béatitude. Je me sentais soulevé vers Dieu comme une feuille par le vent. (Bordeaux, $1916: 116-117)$

C'est ici l'archétype même de la mort du juste, délivrance presque joyeuse qui résonne en écho parfait à l'antique Dulce et decorum est pro patria mori du poète Horace. C'est la mort calme du chevalier, des braves à Roncevaux (Ariès, 1975 : 21-23) dans l'abandon total à Dieu, sans ressentiment ni révolte. Cet exemple permet aussi la mise en place d'un modèle à suivre : la conduite de cet officier lui a valu ce bref instant de félicité et on apprend en effet au début de ce récit - d'ailleurs qualifié d'« enseignement » - que le capitaine en question et son ordonnance, «tous deux Bretons, tous deux croyants, (...) avaient communié le matin ensemble avant de partir à l'assaut. » (Bordeaux, 1916: 116). En liant sa foi à la défense de la patrie, le chrétien peut ainsi s'assurer une paix semblable, une bonne mort, tout comme celle qu'aurait pu connaître le capitaine D...

L'exercice rhétorique auquel se livre Bordeaux avec ces deux évocations s'apparente par conséquent à une forme d'exorcisme et délivre un message aussi clair que rassurant: quelle que soit la façon dont vous mourrez au champ d'honneur, vous n'avez rien à craindre. De fait, et malgré leurs différences, chacun de ces récits dépeint une mort héroïque, au sens où la définit Guyénot : "La mort du héros a généralement une portée exemplaire ; il doit avoir réussi sa sortie, c'est-à-dire son entrée dans l'autre monde. La façon dont il a trépassé doit être assimilable à une mort rituelle et sacrificielle.» (Guyénot, 2011: 37). Chez Bordeaux, le spectacle de la mort a donc avant tout une vocation didactique et allégorique.

$\mathrm{Au}$ Moyen Âge, les danses macabres n'étaient pas que de simples représentations destinées à impressionner les fidèles ; à l'image du célèbre Dit des trois morts et des trois vifs, elles s'inscrivent dans une tradition morale et visent à susciter une action particulière, parfois la requête d'un sacrement : « On pourrait encore citer comme modèle contribuant à la genèse des danses macabres le sermon de Carême ou les poèmes de pénitence, dont 
les lamentations sur les péchés commis aboutissent à la confession et à des actes de contrition. » (Wetzel, Sziraky, $2011: 17$ )

Il en va exactement de même avec l'image de la mort dans La Chanson de Vaux, image qui ne doit en aucun cas être le prétexte d'une remise en question du bien-fondé de la guerre et encore moins pour en illustrer l'absurdité ; elle doit au contraire pousser à l'effort, au dépassement, comme l'atteste la dernière phrase du chapitre : «Le sentiment de la mort ne nous demande pas en ce moment des méditations, mais de l'action... »

Avec cette dernière exhortation, nous sommes une fois de plus très éloignés de la rhétorique de Barbusse, chez qui le spectacle de la mort doit entrainer non pas une action, mais une défection, une fuite loin de l'obéissance aveugle. L'horrible anéantissement de l'existence est un appel qui trouve dans les cris de révolte des vivants, vers la fin du roman, une réponse adéquate :

- Ce serait un crime de montrer les beaux côtés de la guerre, murmura un des sombres soldats, même s'il y en avait!

(...) En réalité, le sacrifice des soldats est une suppression obscure. Ceux dont la multitude forment les vagues d'assaut n'ont pas de récompense. Ils courent se jeter dans un effroyable néant de gloire. On ne verra jamais accumuler même leurs noms, leurs pauvres petits noms de rien. (Barbusse, $1917: 235$ )

Chez Bordeaux, la présence de la mort traduit au contraire un encouragement à poursuivre le combat et alors qu'elle déshumanise chez Barbusse - au sens propre comme au sens figuré - elle devient dans La Chanson de Vaux une porteuse d'humanité, comme l'affirme l'auteur dans le premier chapitre des Captifs délivrés, lorsqu'il rend hommage à “tous les morts (...) qui resteront à jamais couchés dans cette terre de Meuse dont leur chair aura fait une terre humaine. » (Bordeaux, 1917 : 30)

Finalement, en dépit de ces divergences considérables, la représentation de la mort occupe chez ces deux auteurs une place identique et reflète leur orientation idéologique ; puisqu'elle constitue une réalité de chaque instant et qu'elle incarne l'aspect le plus tragique de la guerre, il est somme toute logique qu'elle soit l'axe essentiel de toute considération pacifiste ou patriotique.

\section{La parole de l'ennemi}

Bien conscient du fait que cette sublimation de la mort et les discours hérö̈ques qui l'accompagnent ne sont pas suffisants, Henry Bordeaux consacre le chapitre suivant à démontrer les conséquences réelles d'un tel engagement guerrier et cherche à illustrer son impact dans les faits, sur le terrain. "Quelle existence contraignons-nous le Boche à mener en face de nous? » (Bordeaux, 1916:120) demande-t-il ainsi en préambule à une vaste collection de lettres saisies sur les morts et les prisonniers allemands. Cette démarche fait immanquablement penser à celle de Joseph Bédier, qui au début du conflit, avait reçu pour mission de publier des documents rassemblés dans des conditions semblables, non pour avoir un témoignage sur la vie quotidienne de l'ennemi mais pour mettre à jour ses ambitions destructrices et ses nombreux crimes de guerre. Le projet déboucha en janvier 1915 sur une brochure, Les Crimes allemands d'après les témoignages allemands, "aussitôt traduite par le Ministère de la Guerre dans les principales langues d'Europe et diffusée dans tout le continent » (Corbellari, 1997 : 426), bientôt suivie par un second volet intitulé Comment l'Allemagne essaie de justifier ses crimes : les deux publications seront ensuite reprises et prolongées sous le titre Les Violations des lois de la guerre par 
l'Allemagne et connaîtront aussi bien un «immense retentissement » qu'une "marée de polémiques » (Corbellari, $1997:$ 427).

Bien qu'ayant un objectif différent, l'entreprise de Bordeaux découle de la même logique : recourir à une source première, qui échappe à toute volonté de distorsion ou de dissimulation. En outre, le fait qu'il s'agisse de lettres souvent adressées à des membres de la famille ou des proches apparaît comme une indiscutable caution de sincérité. Mais la sélection des lettres, les commentaires qui les accompagnent, l'alternance entre discours direct et anecdotes rapportées ainsi que le choix des thèmes abordés contribuent bien sûr à donner une vision très subjective des évènements.

Comme le fait remarquer Alain Corbellari au sujet de Bédier :

L'Allemand est l'ennemi et ne saurait, au premier degré, susciter que la haine ; il est l'agresseur et toute possibilité de l'absoudre est d'entrée de jeu exclue. Cependant, il n'est pas indifférent que le décryptage soit le fait d'un simple fonctionnaire ou d'un éminent philologue ${ }^{6}$. Se pose ici, radicalement, la question de la méthode et donc du regard qui doit guider la lecture. (Corbellari, 1997 : 431).

Dans le cas d'Henry Bordeaux, cette question du regard se pose exactement de la même manière : l'écrivain ne sera-t-il en effet pas porté à choisir des textes où résonnent certains accents romanesques? Il est en tout cas certain que la plupart des lettres sélectionnées participent à une mise en scène tragique de la défaite allemande - que ce soit par le style pathétique ou la tonalité pacifiste - et tout concourt à dresser un tableau épouvantable de la situation sans toutefois susciter trop de pitié chez le lecteur. Alors que la tâche de Bédier était facilitée par le caractère abject des crimes évoqués, Bordeaux doit veiller à maintenir un difficile équilibre entre deux tendances : humaniser l'ennemi pour rendre crédibles ses souffrances tout en conservant intact le ressentiment vis-à-vis de l'envahisseur.

Pour commencer, le paratexte mérite notre attention : on relèvera par exemple l'en-tête d'une lettre datée du 20 mars 1916 et mentionnant Strassburg (Prusse) comme lieu de rédaction... Difficile de trouver symbole plus éloquent, surtout lorsqu'on connaît le rôle capital joué par les provinces perdues dans l'idéologie de la Revanche! Les textes de Bédier sur les crimes allemands n'ont-ils d'ailleurs pas été publiés par Berger-Levrault, une maison d'édition alsacienne? (Corbellari, $1997: 427$ )

Afin de parfaire sa diabolisation de l'Allemand, Bordeaux va mêler récits dramatiques et clichés germanophobes : un exemple caractéristique de cette double tonalité se retrouve dans une lettre non datée, retrouvée sur un blessé allemand capturé en avril. Le soldat en question y démontre un profond pessimisme, avant d'enchaîner sur des considérations nettement plus terre-à-terre :

Et nous n'avons pas encore été ici devant l'ennemi, mais nous y allons demain et ce n'est pas une petite affaire. J'ai déjà abandonné tout espoir de vous revoir. Celui qui sortira d'ici entier pourra remercier Dieu. J'ai reçu votre paquet, ainsi que je vous l'ai décrit par carte postale et je l'ai consommé immédiatement, car je ne savais pas si je pourrais encore le faire plus tard. (Bordeaux, 1916 : 126)

Cette lettre qui « mêle la religion et l'usage de comestibles » (Bordeaux, 1916:126) est la parfaite illustration de ce que les auteurs nationalistes considéraient comme un trait caractéristique de la pensée allemande, à savoir l'intérêt quasi obsessionnel pour tout ce qui touche à la nourriture. L'accusation se retrouvera sous la plume de Barrès qui, dans sa trilogie des Bastions de l'est, stigmatise l'architecture germanique de Metz avec de semblables rapprochements : "Au sortir de la gare, on tombe dans un quartier tout neuf où des centaines de maisons chaotiques nous allèchent d'abord par leur couleur café au 
lait, chocolat ou thé, révélant chez les architectes germains une prédilection pour les aspects comestibles. » (Barrès, $1918: 8$ )

Par extension, l'Allemand est donc fréquemment dépeint comme un pillard sans cesse affamé et surtout incapable de toute pensée supérieure, ce qui vient renforcer l'idée d'une guerre juste menée par la civilisation contre des barbares matérialistes. De même, Bordeaux ne manque pas l'occasion de souligner que l'ennemi est puni par où il pèche, une autre lettre affirmant qu' « il faut aller chercher le repas à 3 kilomètres en arrière aux cuisines roulantes, et là aussi, il y a danger de mort. Nous avons tous les jours des tués et des blessés parmi ceux qui vont chercher le repas... » (Bordeaux, 1916 : 130)

Mais les Allemands ne se contentent bien sûr pas de songer au ravitaillement: sanguinaires et cruels, ils sont de surcroît moqueurs et cyniques, comme l'atteste une anecdote, au demeurant assez imprécise, où un " permissionnaire a vu passer un convoi de prisonniers français dans une gare allemande: des femmes se moquaient d'eux et les insultaient. »

40 La réplique d'un soldat français est sans appel: «Femmes allemandes, ne riez pas. Nous sommes prisonniers, c'est vrai, mais il y a devant Verdun des Allemands étendus par tas aussi hauts que ça.» (Bordeaux, 1916 : 122-123)

41 Voilà qui suffit à faire taire les rieuses, confirmant de fait que l'arrogance allemande ne peut être contrée que par la violence, seul langage compris de l'ennemi. Ce postulat sert d'ailleurs de justification à l'effort de guerre français, dont l'ardeur est maintes fois mise en évidence dans les extraits choisis. Jugeons-en plutôt à l'aide de ces quelques exemples, démontrant tous la vaillance de l'adversaire: un combattant capturé le 9 mars affirme que «les hommes se sauvent et se défendent dans les maisons où les Français les massacrent à coup de grenades. Les prisonniers ont l'impression que toute la $13^{\mathrm{e}}$ compagnie a été exterminée. » (Bordeaux, 1916: 121), tandis qu'un autre explique que « le reste [de sa compagnie] a dû être anéanti, les feux de barrage empêchant de fuir et de regagner les tranchées de départ. Ils ont d'ailleurs vu tomber la plupart de leurs camarades. » (Bordeaux, 1916 : 122). Quelques pages plus loin, dans un effet de gradation particulièrement efficace, les propos toujours plus elliptiques trahissent un désespoir absolu : « Le 24 mars 1916, devant le fort de Vaux. - Je n'ai pas besoin d'en écrire d'avantage. Tout le reste se comprend. Je veux cependant avoir de l'espoir. C'est amer ! bien amer ! Je suis encore si jeune! A quoi bon? Que sert de prier, de supplier? Les obus! les obus!» (Bordeaux, 1916: 125)

42 Mais les lettres sélectionnées ne se limitent pas aux simples témoignages de soldats; Bordeaux a pris soin d'y ajouter des correspondances envoyées par des familles allemandes à leurs proches au front, afin d'avoir un aperçu de la vie civile. Plusieurs documents font état de conditions catastrophiques : faim et misère sont le quotidien de l'arrière et la population n'est guère mieux lotie que l'armée.

43 Cependant, ce châtiment, aussi dur soit-il, apparaît comme mérité et plusieurs passages laissent sous-entendre que les souffrances allemandes s'apparentent plus à une punition divine qu'à la conséquence d'opérations militaires : la conclusion apportée par l'auteur au terme de ce long catalogue se situe clairement dans une perspective eschatologique :

Que le soldat allemand qui se bat à Verdun connaisse par surcroît l'insécurité matérielle de ceux qu'il a laissés en arrière, c'est une trop juste punition de l'abominable fléau déchaîné par une nation, toute entière enivrée de sa force, qui ricanait quand Paris avait faim en 1870 et qui a voulu organiser la guerre de terreur, et c'est une brûlure de plus dans son enfer. (Bordeaux, 1916 : 133) 
44 la guerre de Cent Ans, cherche à conférer une dimension spirituelle aux combats temporels pour les justifier ou les glorifier. Durant la guerre franco-prussienne de 1870, cette tendance fut surtout illustrée par Paul Déroulède, qui mêla considérations métaphysiques et rhétorique belliciste ; ainsi, dans ses Nouveaux Chants du soldat, parus en 1875, il place certains de ses poèmes à l'époque biblique ou médiévale, donnant ainsi à la Revanche une dimension sacrée et assimilant la France à un peuple élu combattant païens et Sarrasins. Dans le contexte passionné de la Première Guerre mondiale, Léon Bloy reprendra le même genre d'arguments, signant en 1915 Jeanne d'Arc et l'Allemagne, pamphlet où il n'hésite pas à qualifier l'Allemagne protestante de nation hérétique que doit combattre la France catholique...

Pures constructions subjectives, ces clichés tendent en définitive à établir des vérités universelles et immuables: lorsque Bordeaux décrit l'Allemand, il ne songe pas au Germain, au Prussien ou au soldat ennemi, mais à un type d'Allemand éternel dont les caractéristiques traverseraient l'Histoire. Cherchant à conforter les soldats dans leurs convictions, il veut bâtir des certitudes et affermir l'idée d'une guerre juste et sacrée, menée au nom des plus hauts principes.

Pour conclure cette analyse, je souhaiterais poser une dernière question : le point de vue de Bordeaux a-t-il-changé avec les années? Il est certes difficile de le dire, mais dans un recueil de souvenirs daté de 1960, l'écrivain affirmait que la guerre avait laissé sur lui « des traces profondes », avant d'énumérer les titres de plusieurs de ses romans, parus entre 1919 et 1947, et abordant des thématiques liées au conflit (Bordeaux, 1960 : 83). Et si la plupart de ces textes reprennent les idées exposées dans la Chanson de Vaux-Douaumont sans les désavouer, c'est néanmoins avec plus d'émotion ${ }^{7}$ : les accents épiques sont toujours présents, mais on ressent parfois une certaine tristesse à l'évocation de "cette geste de Verdun et de la bataille de France où des cœurs humains, répandant leur sang pour le pays, triomphèrent de l'épouvante et de l'horreur de la guerre... » (Bordeaux, $1960: 83)$.

\section{BIBLIOGRAPHIE}

BORDEAUX, Henry (1924 [1916]). La chanson de Vaux-Douaumont *, Les derniers jours du Fort de Vaux (9 mars-7 juin 1916). Paris : Plon.

BORDEAUX, Henry (1917). La chanson de Vaux-Douaumont **, Les captifs délivrés (21 octobre- 3 novembre 1916). Paris : Plon.

ARIÈs, Philippe (1975). Essai sur l'histoire de la mort en Occident, Du Moyen Âge à nos jours. Paris :

Éditions du Seuil.

BARRÈs, Maurice (1916). Dix jours en Italie. Paris : Éditions Georges Crès \& Cie.

BARRÈs, Maurice (1940 [1918]). Colette Baudoche, Histoire d'une jeune fille de Metz. Paris : Plon.

BARBUSSE, Henri (1917). Le Feu. Paris : Flammarion.

Carnets, Deuxième série - 5 | 2015 
BORDEAUX, Henry (1960). L'amour de la terre et de la maison. Paris : Wesmaer-Charlier.

BUTTIN, Anne (1990). Henry Bordeaux, romancier savoyard. Chambéry : Mémoires et Documents XCII, Société Savoisienne d'Histoire et d'Archéologie.

CÉLINE (2006 [1952]). Voyage au bout de la nuit. Paris : Gallimard.

CENDRARS, Blaise (2013 [1946]). La main coupée. Paris : Denoël.

CORBELLARI, Alain (1997). Joseph Bédier, écrivain et philologue. Genève : Droz.

KAEMPFER, Jean (1998). Poétique du récit de guerre. Paris : José Corti.

LE NAOUR, Jean-Yves (2012). 1914, La grande illusion. Paris : Perrin.

HARDIER, Thierry (2014 [2012]). « Mourir sur le Chemin des Dames : le traitement des corps, les sépultures et monuments pendant la guerre ", in OFFENSTADT, Nicolas (dir.). Le Chemin des Dames. Paris : Tempus Perrin, pp. 347-375.

GUYÉNOT, Laurent (2011). La mort féerique, Anthropologie du merveilleux, XII -XVe siècle. Paris :

Gallimard NRF.

JOLY, Bertrand (2012 [2004]). “Le souvenir de 1870 et la place de la Revanche ", in AUDOIN-ROUZEAU, Stéphane \& BECKER, Jean-Jacques (dir.). Encyclopédie de la Grande Guerre. Tome I. Paris : Tempus Perrin, pp. 137-159.

SCHIVELBUSCH, Wolfgang (2006 [2001]). La cultura dei vinti. Bologna : il Mulino.

TOUBERT, Pierre \& ZINK, Michel (2009). Moyen Âge et Renaissance au Collège de France. Paris : Fayard. WETZEL, René, SZIRÀKY, Anna (2011). Danse Macabre : Incunable allemand, Mayence, Jacob Meydenbach, vers 1490. Paris : Presses Universitaires de France.

\section{NOTES}

1. Philippe Pétain, colonel d'infanterie proche de la fin de carrière en 1914, sera ensuite nommé général et prendra la tête de la II ${ }^{\mathrm{e}}$ armée en juin 1915. Envoyé à Verdun le 25 février 1916, il va y acquérir une immense popularité et sera nommé Maréchal en décembre 1918.

2. Le paratexte éditorial des Captifs délivrés nous apprend ainsi que mille exemplaires ont été offerts à l'armée par les éditeurs et l'auteur; ce dernier a d'ailleurs renoncé à ses droits sur cinq mille autres exemplaires afin de reverser les bénéfices à différentes associations d'entraide pour les familles de soldats.

3. Sur cette question, voir par exemple la leçon inaugurale de Gaston Paris au Collège de France, le 8 décembre 1870 : La Chanson de Roland et la nationalité française, in Toubert, Pierre \& Zink, Michel (2009), Moyen Âge et Renaissance au Collège de France, Paris : Fayard, pp.105-118.

4. Souvenons-nous de la fameuse «nuit du Moyen âge » évoquée en 1902 par Léon Bloy dans son Exégèse des Lieux Communs...

5. Henry Bordeaux consacra de nombreux textes à cette période, d'ailleurs souvent élogieux : parmi ceux-ci, on peut relever Le mystère du roi saint Louis ou encore La nouvelle croisade des enfants. 6. Son statut de médiéviste a sans doute aussi contribué à façonner son point de vue...

7. C'est particulièrement le cas dans La lumière au bout du chemin (1948), roman qui évoque un jeune couple séparé par la guerre. 


\section{RÉSUMÉS}

Alors que beaucoup d'auteurs ont dépeint la Première Guerre mondiale comme un affreux carnage dénué de sens, certains lui ont conféré une signification spirituelle et tentèrent de la présenter comme un combat pour les plus hautes valeurs de l'humanité. La Chanson de VauxDouaumont, par l'auteur catholique français Henry Bordeaux est une illustration parfaite de cette tendance : décrivant les combats de 1915 dans les environs de Verdun pour le contrôle des forts de Vaux et de Douaumont, il les montre comme les étapes d'une guerre sainte et compare la victoire française à la revanche de Charlemagne après Roncevaux. Cette contribution se concentrera sur les trois principaux arguments utilisés par Bordeaux dans ce processus : ses références au Moyen Âge, ses commentaires sur la mort des soldats au champ d'honneur et le portrait qu'il dresse des Allemands en tant qu'ennemis désespérés mais féroces, combattant pour un empire oppressif et cruel devant être terrassé par la Vertu de la France et de ses alliés.

While many authors used to depict the World War I as a senseless and gruesome carnage, some gave it a spiritual meaning and tried to show it as a fight for the highest values of humanity. $\mathrm{La}$ Chanson de Vaux-Douaumont, by French catholic writer Henry Bordeaux is a perfect illustration of this tendency: describing the fights around Verdun in 1915 for the control of the Fort de Vaux and the Fort of Douaumont, he presents them as parts of a holy war, comparing the French victory to Charlemagne's revenge after the last stand of Roland at Roncevaux. This contribution will focus on the three main arguments used by Bordeaux in this process: his references to the Middle Ages, his comments about the death of the soldiers on the battlefield, and the depicting of the Germans as desperate yet feral enemies, fighting for an oppressive and cruel empire who needs to be defeated by the Virtue of the French and their allies.

\section{INDEX}

Mots-clés : Première Guerre mondiale, Verdun, Moyen Âge, mort, Roland

Keywords : World War I, Verdun, Middle Ages, death, Roland

\section{AUTEUR}

\section{OLIVIER WICKY}

Université de Lausanne

olivier.wicky@unil.ch 